\title{
Editorial Introduction to the Artificial Life 14 Conference Special Issue
}

\author{
Hiroki Sayama ${ }^{1}$, John Rieffel², Sebastian Risi³, René Doursat ${ }^{4} \&$ Hod Lipson $^{5}$ \\ ${ }^{1}$ Center for Collective Dynamics of Complex Systems, and Department of Systems Science and Industrial Engineering, \\ Binghamton University, State University of New York, Binghamton, NY 13902-6000, USA. \\ ${ }^{2}$ Department of Computer Science, Union College, Schenectady, NY 12308, USA. \\ ${ }^{3}$ Robotics, Evolution, and Art Lab, IT University of Copenhagen, Rued Langgaards Vej 7, 2300 Copenhagen, Denmark. \\ ${ }^{4}$ Informatics Research Centre, School of Computing, Mathematics, \& Digital Technology, \\ Manchester Metropolitan University, Manchester, M15 6BH, UK. \\ ${ }^{5}$ Department of Mechanical Engineering, Columbia University, New York, NY 10027, USA. \\ sayama@binghamton.edu,rieffelj@union.edu, sebastian.risi@gmail.com,r.doursat@mmu.ac.uk, hod.lipson@columbia.edu
}

This special issue displays some of the best articles presented at the Fourteenth International Conference on the Synthesis and Simulation of Living Systems (ALife 14), which was held in New York, USA, on July 30-August 2, 2014 (http://alife2014.alife.org/). ALife 14 was the fourteenth convening of ALife, one of the two largest conference series focusing on the study of artificial life, alternating with ECAL, the European edition.

ALife 14 attracted a total of 203 submissions, solicited in two formats: full article (up to 8 pages) and extended abstract ( 2 pages). Both types were evaluated through multiple peer reviews by the Program Committee. As a result, 102 submissions were accepted for oral presentations and 62 for poster presentations. These articles and abstracts were published in the open-access conference proceedings available from MIT Press' website (https://mitpress.mit.edu/index.php?q=books/artificial-life-14). The conference program was full of scientific, professional, and creative activities, including five superb keynote talks, twenty parallel sessions covering a wide variety of topical areas, a very well-attended poster session with a plenary "Poster Blitz Movie Show," nine workshops, six tutorials, the First Summer School of the International Society for Artificial Life (ISAL) with four lectures, a science visualization competition, and a career-advising luncheon for postdocs and graduate students. These were four vibrant, intellectually stimulating days in Manhattan.

The contributions included in this special issue were selected, by the Organizing Committee and the Best Paper Committee, from the full articles presented orally at the ALife 14 conference, based on their peer review scores and the quality of their presentations. The Best Paper and the Best Student Paper of ALife 14 (http://blogs.cornell.edu/alife14nyc/best-paperposter-awards/) were also included in this special issue. Authors submitted an extended version of their conference manuscript, which went through another round of peer review and revision for journal publication. As a result, there are eight articles included in this special issue. The first four are about artificial chemistry: two discussing abstract models and two addressing real biochemistry. The last four articles are about evolution, two of them also covering morphological adaptation and computing. Their contents are summarized below.

The first article, "Complex Autocatalysis in Simple Chemistries," received the Best Paper award at ALife 14. In this article, Virgo, Ikegami, and McGregor present simple mass-action-kinetics-based artificial chemistry models and demonstrate that, even in a thermodynamically reversible chemical reaction network, complex autocatalytic cycles can emerge when certain direct reactions are prohibited. Complex nonlinear dynamics, such as bistability, arising in their simple models may offer new insight into the origins of life.

In "Exploring the Space of Viable Configurations in a Model of Metabolism-Boundary Co-construction," Agmon, Gates, Churavy, and Beer propose a spatial artificial chemistry model in which autopoietic interaction between boundary formation and metabolic reactions inside it allows stable cellular structures 
to emerge. They systematically examine the robustness and variability of these cellular structures by subjecting them to global or local perturbations, revealing a transition network of viable configurations.

In "Computational Design of a Circular RNA with Prionlike Behavior," Badelt, Flamm, and Hofacker present a novel theoretical chemistry method to computationally design RNA molecules that have desired properties (energy landscapes), using their software tool called the Vienna RNA package. They demonstrate the effectiveness of the proposed method by using it to design self-replicating RNA molecules that show conformational self-replication, just like the self-replication of prions.

In "Compartmentalization of an all-E. coli Cell-Free Expression System for the Construction of a Minimal Cell," Caschera and Noireaux present an experimental study on the effects of compartmentalization of their cell-free transcription-translation system extracted from E. coli. They show that, when encapsulated in cellsized lipid vesicles, this system exhibits large fluctuations in gene expression levels, possibly due to heterogeneity in DNA template encapsulation.

The next article, "An Informational Study of the Evolution of Codes and of Emerging Concepts in Populations of Agents," was the recipient of the Best Student Paper award at ALife 14. In this article, Burgos and Polani study an agent population model in which no shared code is initially available for agents' communication and the sources of information are not distinguishable to each agent. Using evolutionary optimization, they demonstrate that a universal code can emerge if the population is well mixed, while multiple distinct codes may result in spatially structured populations. Moreover, "blind" agents can develop concepts about the environment using information coming from other agents.

In "A General Statistical Method for Identifying Adaptations by Parameterizing Trait Space," Blount proposes a formal method to detect adaptation by mapping the traits of evolving organisms to a 3D metric space that consists of variation, heritability, and differential fitness. This method does not require a priori specification of what kind of function or purpose the adaptation possesses. As a proof of concept, the calculation of differential fitness is demonstrated through the application of the proposed method to Packard's Bugs system.

In "Population and Evolutionary Dynamics based on Predator-Prey Relationships in a 3D Physical Simulation," Ito, Pilat, Suzuki, and Arita present a virtual ecosystem of 3D morphological creatures like Sims' blockies, where both ecological and evolutionary dynamics develop simultaneously and spontaneously via coevolution of predator and prey populations. Computer simulations show the emergence of short-term (ecological) and long-term (evolutionary) dynamics occurring in two different regimes of time scales, as well as their mutual interactions.

Finally, in "Active Shape Discrimination with Compliant Bodies as Reservoir Computers," Johnson, Philippides, and Husbands propose to use a randomly generated mass-spring-damper (MSD) network embedded in an agent's body as a "morphogenetic computation" device, which is a physical counterpart of neural-network-based reservoir computing. They demonstrate, through evolutionary search, that such an MSD network can be used successfully as a reservoir computer to control the agent when catching objects of a certain shape while avoiding others.

We thank all the authors of the above articles for their excellent contributions, which nicely illustrate the current state of the art of artificial life. We would also like to thank the following people on the Best Paper Committee of ALife 14, who played a crucial role in evaluating numerous articles and selecting the best ones to be included in this volume:

- Chair: Luis Rocha

- Co-chair: Takashi Ikegami 
- Committee members: Joshua Auerbach, Lola Cañamero, Dominique Chu, Sylvain Cussat-Blanc, Stéphane Doncieux, Dusan Misevic, Susan Stepney, Sebastian von Mammen

The success of the ALife 14 conference was due to all who helped and participated in this event, including the Program Committee members, the five keynote speakers (John H. Conway, Lee Cronin, Naomi Leonard, Jesse Louis-Rosenberg, and Karl Sims), the organizers of workshops and tutorials, local organization staff, and the following sponsors:

- US National Science Foundation

- MIT Press

- Cornell University

- Thomas J. Watson School of Engineering and Applied Science at Binghamton University

- Wolfram Research

- Mary Ann Liebert, Inc. Publishers

Finally, we would like to give special thanks to Mark Bedau, the Editor-in-Chief of the Artificial Life journal, and Linda Reedijk, the editorial assistant, for their help, encouragement, and support in this special issue. 